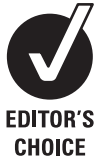

${ }^{1}$ Centre for Paediatric Pharmacy Research, School of Pharmacy, London, UK; ${ }^{2}$ Paediatric Infectious Diseases Unit, St George's Hospital, London, UK ${ }^{3}$ MRC Centre of Epidemiology for Child Health, Institute of Child Health, London, UK

${ }^{4}$ Department of Primary Care and Social Medicine, Imperial College London, London, UK; ${ }^{5}$ Department of Healthcareassociated Infection and Antimicrobial Resistance, Centre for Infections, Health Protection Agency, London, UK

\section{Correspondence to:}

Nikos Spyridis, Paediatric Infectious Diseases Unit, St George's Hospital, Blackshaw Road, London SW17 00T, UK n.spyridis@sgul.ac.uk

Accepted 2 December 2008 Published Online First 9 December 2008

\title{
Changes in clinical indications for community antibiotic prescribing for children in the UK from 1996 to 2006: will the new NICE prescribing guidance on upper respiratory tract infections just be ignored?
}

\author{
P L Thompson, ${ }^{1}$ N Spyridis, ${ }^{2}$ M Sharland, ${ }^{2}$ R E Gilbert, ${ }^{3} S$ Saxena, ${ }^{4}$ P F Long, ${ }^{1}$ \\ A P Johnson, ${ }^{5}$ I C K Wong ${ }^{1}$
}

\section{ABSTRACT}

Objective: To analyse changes in clinical indications for community antibiotic prescribing for children in the UK between 1996 and 2006 and relate these findings to the new NICE guidelines for the treatment of upper respiratory tract infections in children.

Study design: Retrospective cohort study.

Method: The IMS Health Mediplus database was used to obtain annual antibiotic prescribing rates and associated clinical indications in 0-18-year-old patients between 1 January 1996 and 31 December 2006 in the UK.

Results: Antibiotic prescribing declined by $24 \%$ between 1996 and 2000 but increased again by 10\% during 20032006. Respiratory tract infection was the most common indication for which an antibiotic was prescribed, followed by "abnormal signs and symptoms", ear and skin infections. Antibiotic prescriptions for respiratory tract infections have decreased by $31 \%(p<0.01)$ mainly because of reduced prescribing for lower respiratory tract infections $(56 \%$ decline, $p<0.001)$ and specific upper respiratory tract infections including tonsillitis/pharyngitis (48\% decline, $p<0.001)$ and otitis $(46 \%$ decline, $p<0.001)$. Prescribing for non-specific upper respiratory tract infection increased fourfold $(p<0.001)$. Prescribing for "abnormal signs and symptoms" increased significantly since 2001 (40\% increase, $\mathrm{p}<0.001$ ).

Conclusion: There has been a marked decrease in community antibiotic prescribing linked to lower respiratory tract infection, tonsillitis, pharyngitis and otitis. Overall prescribing is now increasing again but is associated with non-specific upper respiratory tract infection diagnoses. General practitioners may be avoiding using diagnoses where formal guidance suggests antibiotic prescribing is not indicated. The new NICE guidance on upper respiratory tract infections is at risk of being ignored.

The overuse of antibiotics in children is a largely unseen but major public health problem. ${ }^{1}$ The largest volumes of antibiotics are prescribed in primary care $e^{2}$ and are frequently given inappropriately for uncomplicated viral infections of the upper respiratory tract. ${ }^{3}$ Excessive antibiotic use is a risk factor for the development of antibiotic resistance ${ }^{4}$ which can increase morbidity, mortality and health care costs. ${ }^{5}$ In the UK, an overall fall in antibiotic prescribing for both adults and children has been noted from the late 1990s to $2000,{ }^{6}$ but there have been no published data on the specific clinical indications leading to antibiotic prescribing

\section{What is already known on this topic}

- Antibiotic prescribing for children seen in primary care has declined since the late 1990s.

- The majority of antibiotics are given for uncomplicated infections of the upper respiratory tract.

\section{What this study adds}

- Antibiotic prescribing for non-specific upper respiratory tract infections in children seen in primary care increased fourfold between 2003 and 2006.

- Full implementation of the new NICE guidelines on antibiotic prescribing for upper respiratory tract infection would lead to a $17-34 \%$ reduction in prescribing.

- This reduction may not be achieved if general practitioners increasingly avoid using the specific clinical diagnoses contained in the NICE guidelines.

for children in primary care. In this retrospective study we analysed time trends and patterns of condition-specific antibiotic prescribing in primary care for children in the UK between 1996 and 2006. We also attempt to link our results to the recent guidelines from the National Institute for Health and Clinical Excellence (NICE) which recommend delayed or no prescribing of antimicrobials for the management of upper respiratory infections in children and adolescents, ${ }^{7}$ and address whether the new guidelines are likely to reduce antibiotic prescribing in the community.

\section{METHODS}

The IMS Health Mediplus UK database was used to obtain data on the annual incidence of antibiotic prescriptions and the associated indications for patients aged 0-18 years between 1 January 1996 and 31 December 2006 in the UK. This database contains anonymous longitudinal data from approximately 125 computerised UK general practices, providing information on over 1 million 
active patients, which represents approximately $2 \%$ of the UK population. The advantage of the IMS data is that antibiotic prescriptions are directly linked to the clinical indication. Indications are coded via the International Classification of Diseases (ICD), which is a hierarchical coding system used to classify disease, with ICD-10 being the latest revision. For the purposes of this study, ICD-10 codes J03 (acute tonsillitis), J02 (acute pharyngitis) and J00 (acute nasopharyngitis) were grouped together under the indication "acute sore throat", while codes J20 (bronchitis) and J22 (acute lower respiratory infection) were combined under the indication "lower respiratory tract infection". ICD-10 code R (abnormal signs and symptoms) included a number of clinical findings, including fever, cough, skin rash, dysuria or a generally unwell child, which by themselves did not constitute a specific diagnosis. Antibiotic prescribing rates were calculated as the number of antibiotic prescriptions divided by the person-years contributed by children aged $0-18$ years registered with participating practices in the IMS Health database. 95\% Confidence intervals (95\% CI) were calculated using Poisson approximation. For all statistical tests a $p$ value of $<0.05$ was considered statistically significant. None of the variables within the database have changed over the study period. Data analysis was performed using SPSS v 16 (SPSS, Chicago, IL). The study received ethical approval from the Independent Scientific and Ethical Advisory Committee.

\section{RESULTS}

Between 1 January 1996 and 31 December 2006, a total of 982811 children aged $0-18$ years contributed data to the Mediplus UK database, comprising 3106672 child-years of follow-up. Overall, 1482430 antibiotic prescriptions were identified over the study period in 334756 children.

\section{Total prescribing}

Total antibiotic prescribing (fig 1) decreased by $24 \%$ between 1996 (572 prescriptions/1000 child-years, 95\% CI 570 to 574) and 2000 (435/1000 child-years, 95\% CI 433 to 437), with rates remaining fairly stable until 2002 (444/1000 child-years, 95\% CI 442 to 445$)$. Antibiotic prescribing increased by $10 \%$ between 2003 (456/1000 child-years, 95\% CI 454 to 458) and 2006 (508/ 1000 child-years, 95\% CI 506 to 510), with the increase being more marked during the last 2 years of the study. Changes were similar across all age groups.

\section{Overall clinical indications}

Respiratory tract infection was the most common indication for antibiotic treatment, followed by "abnormal signs and

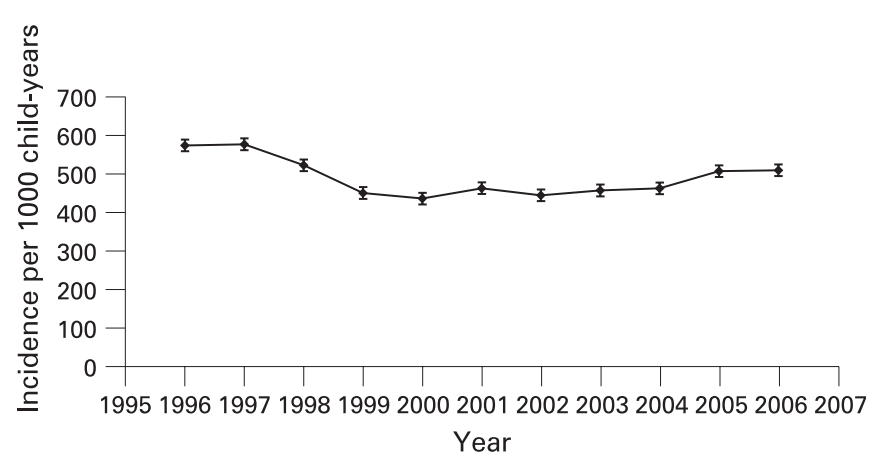

Figure 1 Total antibiotic prescribing in 0-18-year-old children in UK primary care. symptoms", ear and skin infections (fig 2). Over the study period, antibiotic prescriptions for respiratory tract infection decreased by 31\% (241 prescriptions/1000 child-years in 1996 to $166 / 1000$ child-years in 2006, $p<0.01$ ). Prescribing for "abnormal signs and symptoms" declined by 25\% between 1996 and $2000(107 / 1000$ child-years in 1996 to $80 / 1000$ child-years in 2000, $\mathrm{p}<0.009$ ) but then increased by $40 \%$ from 2001 to 2006 $(135 / 1000$ child-years, $p<0.001)$. Prescriptions linked to ear infections (otitis) decreased by 46\% (94/1000 child-years in 1996 to $50 / 1000$ child-years in $2006, p<0.001$ ), while prescriptions for skin infections increased by 74\% (47/1000 child-years in 1996 to $82 / 1000$ child-years in 2006, $\mathrm{p}<0.001)$.

\section{Respiratory tract indication}

A detailed analysis of the upper respiratory tract indication (fig 3) shows that during the study period prescribing for "acute sore throat" (tonsillitis, pharyngitis, nasopharyngitis) declined by almost 48\% (82 prescriptions/1000 child-years in 1992 to 42/ 1000 child-years in 2006, $\mathrm{p}<0.001$ ), while prescribing for nonspecific upper respiratory tract infections increased fourfold (13/ 1000 child-years in 1996 to 50/1000 child-years in 2006, $\mathrm{p}<0.001)$. Prescriptions linked to a lower respiratory tract infection declined by 56\% (92/1000 child-years in 1996 to 40/ 1000 child-years in 2006, $\mathrm{p}<0.001)$.

\section{Prescribing for "abnormal signs and symptoms"}

Prescribing for "abnormal signs and symptoms" has increased significantly since 2001 (40\%) and is currently the second most common indication for antibiotic prescribing in children in primary care. Analysis of the 2006 data (fig 4, latest available data) revealed that almost two thirds of prescribing for "abnormal signs and symptoms" was linked to an "ill defined diagnosis" (73\%).

\section{DISCUSSION}

This is the first study to provide a detailed breakdown of specific indications for antibiotic prescribing in children in UK primary care. The large number of children included in the study and the substantial time frame enables robust examination of prescribing trends, although results are limited by the fact that the diagnoses made were clinical with no microbiological confirmation.

\section{Prescribing indications}

This study has suggested that in primary care prescribing for specific upper respiratory tract infection indications that are more likely to be caused by a viral infection has substantially declined ("acute sore throat" (tonsillitis, pharyngitis) by $48 \%$, otitis by $46 \%$ ). This has been associated with a fourfold increase in prescribing for non-specific upper respiratory tract infections and more recently a $40 \%$ increase in prescriptions for children with "abnormal signs or symptoms". This high rate of prescribing for these non-specific diagnostic groups was responsible for the increase in total prescribing towards the end of the study. It is possible that general practitioners (GPs), in response to ever more guidelines suggesting reducing prescribing for specific upper respiratory tract diagnoses, ${ }^{9}$ have shifted their prescribing to diagnoses where there is less formal guidance. This is happening despite recent data suggesting that the great majority of children with non-specific upper respiratory tract infection symptoms have an underlying viral cause for their illness. Harnden et al ${ }^{3}$ recruited children with non-specific upper respiratory tract infection diagnoses and identified a viral 


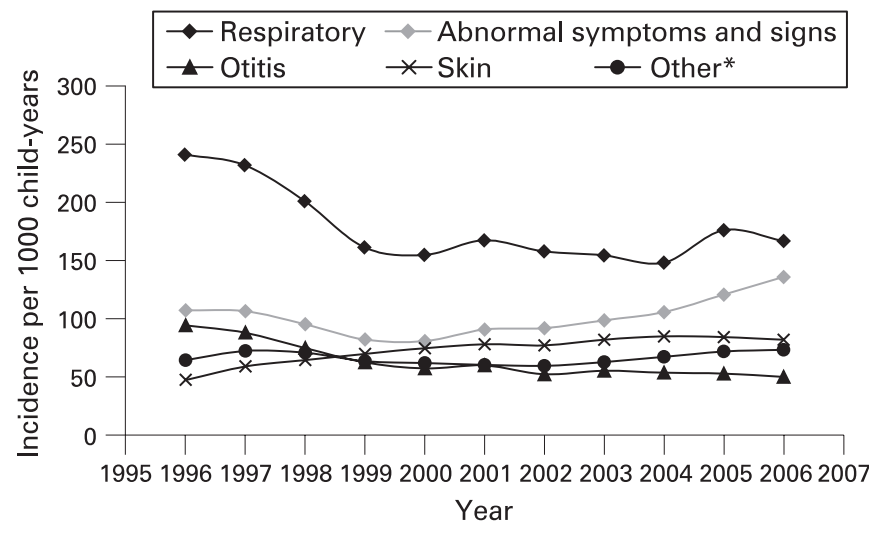

Figure 2 Antibiotic prescribing indications in 0-18-year-old children in UK primary care. *Includes genitourinary infections, metabolic, nutritional and endocrine related infections and infections following contact with health services.

cause in $77 \%$. Children with RSV and metapneumovirus isolated were most likely to receive an antibiotic.

This indicates that perhaps the key decision by the doctor faced with an unwell child is whether to prescribe or not, and that the specific diagnosis is then made secondary to that decision. There is already sufficient evidence to show that prescribing in children is strongly related to parental satisfaction, ${ }^{10}$ communication skills or the way physicians perceive parental expectations. ${ }^{11}$ In this case more guidelines or recommendations may have little or no effect on total antibiotic consumption if individual prescribing habits play the most important role, although GPs should be encouraged not to prescribe antibiotics in children with non-specific signs and symptoms where the diagnosis is in doubt.

\section{The new NICE guidelines}

The evidence base of antibiotic prescribing for upper respiratory tract infections has been recently reviewed by NICE, with new guidance recommending either a delayed or no prescribing policy for five common diagnoses: acute otitis media, acute cough/bronchitis, acute sore throat, acute sinusitis and common cold. There is a strong evidence base that this approach is both safe and effective in reducing antibiotic prescribing within clinical trials. ${ }^{12-14}$ The evidence supporting the guidelines suggests that delayed prescribing can lead to a $63 \%$ reduction in antibiotic use for acute otitis media, $80 \%$ for cough, 31\% for acute sore throat and $46 \%$ for common cold. ${ }^{7}$ Application of these data to our study population would lead to an average $34 \%$ decline in total antibiotic use for a no prescribing policy and a $17 \%$ decline for a delayed prescribing policy.

This shift to non-specific indications may limit the use of guidelines to contain antibiotic prescribing. It is unclear why prescribing is rising again. The first possibility is that GPs are not convinced that a low prescribing rate is safe and not related to adverse outcome. Sharland et $a l^{6}$ have shown that hospital admissions for peritonsillar abscess and rheumatic fever did not increase despite the reduction in antibiotic use in the late 1990s, although they raised concerns about an increase in rates of mastoiditis. Another retrospective cohort study also demonstrated that GPs would need to treat almost 4800 children with otitis media in order to prevent one case of mastoiditis. ${ }^{15}$ This was confirmed by Petersen et $a l^{16}$ who suggested that the overall number of courses of antibiotics needed to prevent one serious complication is over 4000. There are concerns that these studies

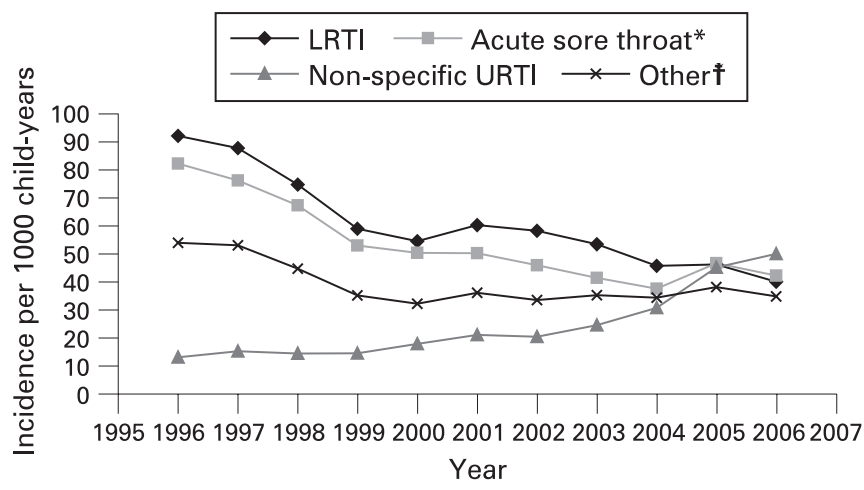

Figure 3 Respiratory indications for antibiotic prescribing in 0-18-yearold children in UK primary care. ${ }^{*}$ Included the indications tonsillitis, pharyngitis and nasopharyngitis. †Included unspecified respiratory disorder, allergic asthma and asthma unspecified. LRTI, lower respiratory tract infection; URTI, upper respiratory tract infection.

may be confounded by indication as unwell children with more systemic symptoms are more likely to have received an immediate prescription instead of a delayed or no prescription. A further possibility is that antibiotics are given for symptom control. Little et a ${ }^{14}$ suggested that the benefit from immediate antibiotic prescription in acute otitis media was limited to symptomatic relief after the first $24 \mathrm{~h}$ when symptoms are already resolving, concluding that delayed prescribing seems a reasonable approach for patients and carers. Meta-analysis of randomised controlled trials shows antibiotic use to treat sore throat, ${ }^{17}$ rhinitis $^{18}$ and acute otitis media ${ }^{19}$ has minimal or no benefit on the clinical outcome. But GPs may be sceptical of this evidence and feel that the individual needs of their local population outweigh any concerns about antibiotic overuse and bacterial resistance. ${ }^{20}$

\section{So where next?}

Firstly, we need more information on the determinants of both high prescribers of antibiotics in primary care and children who are high receivers. Are there specific features of either or both that could lead to a targeted approach? How can we improve the implementation of delayed prescribing in primary care, with the negotiation this requires with families? Secondly, we need more studies on integrating near patient testing for respiratory viruses using multiplex PCR. ${ }^{21}$ This will be too expensive to use in routine clinical practice in the near future, but further clinical studies will be very helpful in demonstrating that in the era of universal conjugate pneumococcal vaccination, a virological diagnosis can probably be made in virtually every child with an

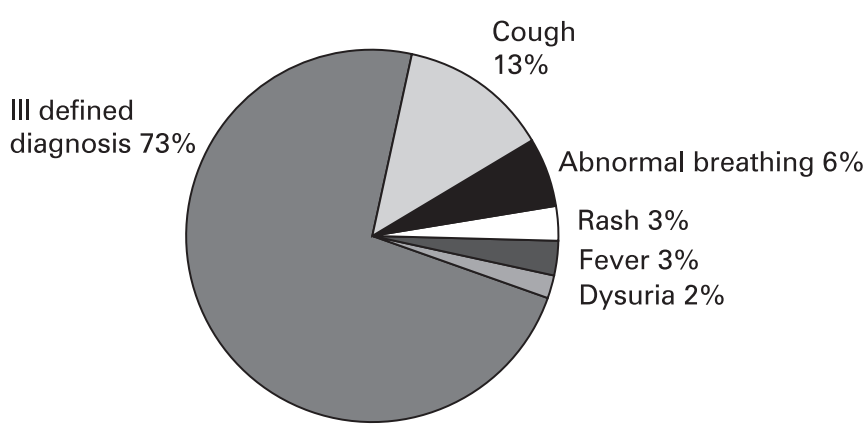

Figure 4 Relative contribution of diagnoses for children with "abnormal signs and symptoms" in 2006. 
upper respiratory tract infection. Thirdly, we have to accept that the current UK national antibiotic campaigns have just not been very successful in engaging the public in decision making and teaching the benefits of self-care. Examples from other European countries suggest that carefully structured national campaigns can be successful especially when electronic media take a leading role. ${ }^{22} 23$ Finally and most importantly, the effects of the NICE guidelines must be actively monitored with regard to antibiotic utilisation, adherence, changes in clinical disease patterns and rare complication rates $^{24}$ so we can clearly demonstrate to both prescribers and the public the safety of the national implementation of both no and delayed antibiotic prescribing advice. The UK in European terms has low antibiotic prescribing rates in primary care. ${ }^{25}$ As we try to reduce this still further, we need to put in place improved systems to monitor the risks and benefits of all antibiotic prescribing in children.

Acknowledgements: We thank IMS for providing the data and all the members of the iCAP group (improving Children's Antibiotic Prescribing).

Funding: NS's post is funded by the European Society for Paediatric Infectious Diseases. ICKW's post was funded by a Department of Health Public Health Career Scientist Award. SS has a post-doctoral award from the National Institute for Health Research (NIHR).

Competing interests: PLT, MS, APJ and ICKW were members of the UK Department of Health's Specialist Advisory Committee on Antimicrobial Resistance (SACAR), paediatrics subgroup. RG and MS are current members of the ARHAl (Antimicrobial Resistance and Healthcare Associated Infection) Committee.

Ethics approval: The study received ethical approval from the Independent Scientific and Ethical Advisory Committee

Author contributions: MS and ICKW had the original idea for the study. PLT extracted the relevant data from the IMS. NS prepared the manuscript in consultation with all the authors.

\section{REFERENCES}

1. Tenover FC, Hughes JM. The challenges of emerging infectious diseases. JAMA 1996;275:300-4.

2. Goossens H, Ferech M, Vander Stichele R, et al. Outpatient antibiotic use in Europe and association with resistance: a cross national data base study. Lancet 2005;365:579-87.

3. Harnden A, Perera R, Brueggemann $A B$, et al. Respiratory infections for which general practitioners consider prescribing an antibiotic: a prospective study. Arch Dis Child 2007:92:594-7.

4. Livermore DM. Minimizing antibiotic resistance. Lancet Infect Dis 2005;5:450-9.

5. Cohen ML. Epidemiology of drug resistance: implications for a post-antimicrobial era. Science 1992;257:1050-5.
6. Sharland M, Kendall H, Yeates D, et al. Antibiotic prescribing in general practice and hospital admissions for peritonsillar abscess, mastoiditis, and rheumatic fever in children: time trend analysis. BMJ 2005;331:328-9.

7. National Institute for Health and Clinical Excellence. Prescribing of antibiotics for self-limiting respiratory tract infections in adults and children in primary care. Clinical guidance CG69, July 2008. Available from http://www.nice.org.uk/Guidance/ CG69 (accessed 10 February 2009).

8. Wong IC, Murray ML. The potential of UK clinical databases in enhancing paediatric medication research. Br J Clin Pharmacol 2005;59:750-5

9. Department of Health Standing Medical Advisory Committee Subgroup on Antimicrobial Resistance. The path of least resistance. Main report. London: DoH, 2000. Available from http://www.advisorybodies.doh.gov.uk/pub/docs/doh/smacrep. pdf (accessed 10 February 2009)

10. Christakis DA, Wright JA, Taylor JA, et al. Association between parental satisfaction and antibiotic prescription for children with cough and cold symptoms. Pediatr Infect Dis J 2005;24:774-7.

11. Mangione-Smith R, McGlynn EA, Elliott MN, et al. The relationship between perceived parental expectations and pediatrician antimicrobial prescribing behavior. Pediatrics 1999;103:711-18.

12. Little $\mathbf{P}$. Delayed prescribing of antibiotics for upper respiratory tract infection. BMJ 2005;331:301-2.

13. Spiro MD, Yen Tay K, Arnold HD, et al. Wait-and-see prescription for the treatment of acute otitis media. JAMA 2006;296:1235-41.

14. Little $\mathbf{P}$, Gould C, Williamson I, et al. Pragmatic randomized controlled trial of two prescribing strategies for childhood acute otitis media. BMJ 2001;322:336-42.

15. Thompson PL, Gilbert RE, Long PF, et al. The effect of antibiotics for otitis media on mastoiditis in children: a retrospective cohort study using the United Kingdom general practice research database. Pediatrics 2009:123:424-30.

16. Petersen I, Johnson AM, Islam A, et al. Protective effect of antibiotics against serious complications of common respiratory tract infections: retrospective cohort study with the UK General Practice Research Database. BMJ 2007;335:982.

17. Del Mar CB, Glasziou PP, Sprinks AB. Antibiotics for sore throat. Cochrane Database Syst Rev 2006;(4):CD000023.

18. Arroll B, Kenealy T. Antibiotics for the common cold and acute purulent rhinitis. Cochrane Database Syst Rev 2005;(3):CD000247.

19. Glasziou PP, Del Mar CB, Sanders SL, et al. Antibiotics for acute otitis media in children. Cochrane Database Syst Rev 2004;(1):CD000219.

20. Simpson SA, Wood F, Butler CC. General practitioners' perceptions of antimicrobial resistance: a qualitative study. J Antimicrob Chemother 2007;59:292-6.

21. Mahony J, Chong S, Merante F, et al. Development of a respiratory virus panel test for detection of twenty human respiratory viruses by use of multiplex PCR and a fluid microbead-based assay. J Clin Microbiol 2007;45:2965-70.

22. Goossens H, Guillemot D, Ferech $\mathrm{M}$, et al. National campaigns to improve antibiotic use. Eur J Clin Pharmacol 2006;62:373-9.

23. Bauraind I, Lopez-Lozano JM, Beyaert A, et al. Association between antibiotic sales and public campaigns for their appropriate use. JAMA 2004;292:2468-70.

24. Sharland $\mathbf{M}$. The use of antibacterials in children: a report of the Specialist Advisory Committee on Antimicrobial Resistance (SACAR) Paediatric Subgroup. J Antimicrob Chemother 2007;60(Suppl 1):i15-26.

25. Muller A, Coenen S, Monnet DL, et al. European Surveillance of Antimicrobial Consumption (ESAC): outpatient antibiotic use in Europe, 1998-2005. Euro Surveill 2007;12(41):pii = 3284. Available from http://www.eurosurveillance.org/ViewArticle. aspx?Articleld $=3284$ (accessed 10 February 2009). 